\title{
AN EFFICIENT ENVIRONMENTAL CHANNEL MODELLING IN 802.11P MAC PROTOCOL FOR V2I
}

\author{
Neelambike $\mathbf{S}^{\mathbf{1}}$ and Chandrika $\mathbf{J}^{\mathbf{2}}$ \\ ${ }^{I}$ Department of Information Science and Engineering, GM Institute of Technology, India \\ ${ }^{2}$ Department of Computer Science and Engineering, Malnad College of Engineering, India
}

\begin{abstract}
Recent development in communication of wireless communication for automobile industry have aided the growth of SITS (Smart Intelligent Transport System) which solves numerous vehicular based communication service concerns like traffic congestion, accidental mishap etc. VANET (Vehicular Ad-hoc Network) a characteristic class of MANET (Mobile ad-hoc Network) which is a fundamental element of SITS in which the moving vehicles inter connected and communicates with each other remotely. Wireless technologies play an important part in assisting both Vehicle to Infrastructure (V2I) and Vehicle to Vehicle (V2V) correspondence in VANET. The existing scheduling technique does not consider the environmental factor which affects the throughput performance and increases packet drop rate which result in degradation of service quality. Here in this work the author propose a RHU (Rural, Highway and Urban) environment model considering the environmental factor. The efficient environmental model algorithm is incorporated into slotted aloha in IEEE 802.11p MAC protocols which aided as a spine for assisting both safety application and non-Safety applications. Experiments are conducted for collision and throughput efficiency for varied traffic load and speed of vehicle. The experimental result shows the proposed environmental model impact on collision and throughput efficiency for varied environment and thus helps improving QoS for VANET application.
\end{abstract}

Keywords:

VANET, V2V, Path Loss, DSRC, WAVE, IEEE 802.11p

\section{INTRODUCTION}

As a basic necessities of a contemporary life, automobile have kept on developing since they were designed in the SIR (Second Industrial Revolution). These days, individuals expect more than automobile quality and unwavering quality. With the ever growing advancement of communication of Information technology (IT), outfitting or providing vehicle with remote or wireless correspondence capacities is relied upon to be the future characteristic necessities. Associated automobiles on the go are composed, coordinated, and proactive, and will make ready for supporting different applications for street wellbeing or safety standard of road infrastructure (e.g., crash recognition, path change cautioning etc.), intelligent and environmental friendly transportation (e.g., Smart Traffic signaling controlling system and efficient public transport system administration). There are two prompt main motivations of conveying wireless connectivity to automobiles. The first is to enhance proficiency, security, and safeness of street transportation infrastructure frameworks. Massive urbanization result an expanding populace of vehicles in huge urban communities, which is in charge result in congestion of road and the outcomes result in gigantic financial expense and ecological issues. It is accounted for that the expense of additional travel time and fuel because of clog in 498 U.S. urban ranges was at that point USD 121 billion in 2011, and delivered amid vehicle traffic clog was 56 billion pounds, contrasted with USD 24 billion and 10 billion pounds in 1982, respectively [4]. Connected vehicle arrangements are exceptionally encouraging to lighten movement congestion of vehicle through smart vehicle control and administration [6], and additionally to enhance the street safety by means of on-board equipped driving assistance and intelligent warning frameworks [8]. The second one is the constantly expanding mobile information interest of clients on street. Provisioning vehicles to the Internet can be imagined not just to take care of the mobile devices information demand [10], additionally improve wellbeing of services related to safety applications, for example, smart tracking and antitheft system [14] and online diagnosis [12].

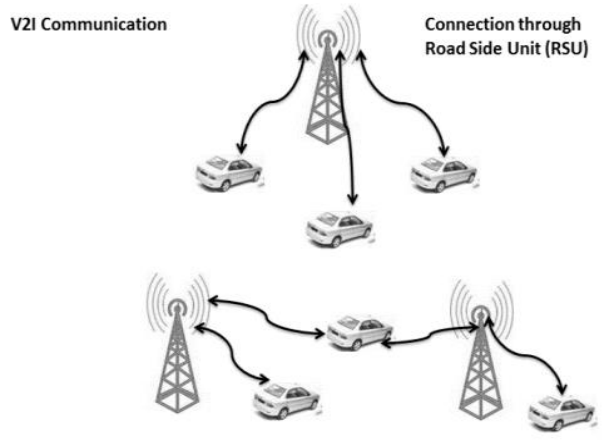

Fig.1. V2I Architecture

VANET depicts a characteristic structure of a MANET in which vehicle or user that are moving behave as a node to transmit packet among the vehicles, or an RSU (Road side unit) which as a communication range of 0.1 to 0.9 kilometers if considering $802.11 \mathrm{p}$. It is intended and designed to cater V2V and V2I association over network without a standard structured infrastructure. It is important to be recognize that the ITS (Intelligent transport system) intentions is to cater road safety and offers a contented tourism or journey experience to motorist [3] [15]. There have been various study initiatives such as CVIS, WAVE (Wireless Access in Vehicular Environments) and ASV (Advanced Safety Vehicle) which is carried out across various countries to make ITS into a practicality. VANETs are intended to provision safety and non-safety application services. Safety related application service such as collision avoidance; pre-crash sensing or lanes changing are intended to reduce accidents on roads by using traffic management application services. NonSafety application services allow users to access various application like browsing internet data, video conferencing and bill payment services etc travelling in vehicle. The noticeable 
variance between non-safety and safety service is that the safety services are proficient of transmitting and handling data in real time [7]. The motorist can avail all classes of application from the nearby RSU effortlessly using wireless technologies [11]. The Fig.1 represent the envisioned architecture of V2I.

There are open challenges and issues as follows. It requires continuous session as it breaks during ESS transition, require many AP's to cover entire city Difficult to achieve seamless roaming, rapid changes in topology, possible frequent disconnections, and environment-introduced multi-path fading: vehicles and stationary objects in big numbers create manifold signal paths, thus causing superposition of numerous copies of the transmitted signal. This leads to inter-symbol interference (ISI) between the successive orthogonal frequency-division multiplexing (OFDM) symbols on a sub-carrier [21], mobilityintroduced Doppler spread: when a moving vehicle receives a wireless signal, its perceived frequency will be Doppler shifted. The shift, which depends on the velocity of a vehicle, can destroy the orthogonality between the adjacent sub-carriers [21]. Does not guarantee good packet reception: there is no approved ideal case that packets have been either perfectly received or not received at all [21], there are weaknesses in particular environments: Environment factors impact significantly the characteristics of DSRC.

Bandwidth varies in both baseband and broadband internet access; however broadband always refer to high speed connection with data transfer rate equal to or more than 256kbps [23], while U.S. Federal Communication Commission(FCC) in 2010 has defined basic Broadband data transfer rate of at least $1 \mathrm{Mbps}$ upstream and $4 \mathrm{Mbps}$ downstream [24]. The minimum bandwidth required to provide broadband basic internet connection on moving vehicles is $384 \mathrm{kbps}$, for both up and down link [25]. Topological structure of persistently changing traffic network has several challenges in providing seamless internet connectivity. There are also requirements of reliability and accessibility between V2X integration even at the extreme vehicular velocity of about $300 \mathrm{~km} / \mathrm{h}$. An efficient handover mechanism to integrate vehicle-land connectivity is still a big challenging issue that remains to be resolved [26]. Though JorgOtt \& Dirk Kutsche, 2004 [20] have managed internet on a moving vehicle using WiFi (802.11b), they faced a number of challenges like 1) transmission range 2) efficient channel handover mechanism 3) high data transfer rate 4) persistent connection and 5) wire equivalent security for suitable applications. Richard et al. 2006, have raised many other issues with respect to the performance of Wi-Fi (802.11b) based internet connection on moving vehicles which includes 1) automation of connection and authentication process 2) application level most common protocol HTTP wherein multi round trips traits needs to be addressed because most web pages required multiple transactions to complete a request, leading to under-use of transfer opportunities 4) Packet loss rate [22]. Many of these challenges like authentication \& association, RF range, Signal Interference, High Data transfer rate, Wire equivalent security, have been adequately addressed in DSRC. Depending upon use of applications various challenges [16] [27] exist using 802.11 based network. One of the challenges to VANETs is the so called grey-zone phenomenon. This phenomenon causes poor or intermediate packet reception that most commonly occurs in the realistic vehicular environments. Additionally, these phenomena should be considered as the normal case for VANET, thereby requiring error-compensation instruments at higher-layers. The other general challenges are Limited transmission capacity, Discontinuous coverage and Vehicles unsystematically arriving/leaving the area. The Table.1 is the list of a few challenges that exist with respect to Transport Layer TCP and network layer IP over DSRC and other generic challenges on 802.11 based vehicular wireless network.

Table.1. Physical network standard overview

\begin{tabular}{|c|c|c|c|c|c|c|}
\hline $\begin{array}{c}\text { Standar } \\
\text { d }\end{array}$ & $\begin{array}{c}\text { Freque } \\
\text { ncy } \\
\text { Band in } \\
\text { GHz }\end{array}$ & $\begin{array}{c}\text { Speed } \\
\text { in Mbps }\end{array}$ & $\begin{array}{c}\text { Indoor } \\
\text { Signal } \\
\text { Range } \\
\text { in meter }\end{array}$ & \begin{tabular}{|} 
Approxim \\
ate \\
Outdoor \\
Signal \\
Range in \\
meter
\end{tabular} & $\begin{array}{c}\text { Mobility } \\
\text { support } \\
\text { in } \approx \mathbf{k m} / \mathbf{h}\end{array}$ & $\begin{array}{c}\begin{array}{c}\text { Suitable } \\
\text { for }\end{array} \\
\text { Outdoor } \\
\text { Network }\end{array}$ \\
\hline \begin{tabular}{|c|} 
Wi-Fi \\
$802.11 \mathrm{a}$
\end{tabular} & $5.1-5.8$ & $25-54$ & 30 & 45 & $40-120$ & Low \\
\hline \begin{tabular}{|c|} 
Wi-Fi \\
$802.11 \mathrm{~b}$
\end{tabular} & $2.4-2.5$ & 11 & 30 & 100 & $40-150$ & Low \\
\hline \begin{tabular}{|c|} 
Wi-Fi \\
$802.11 \mathrm{~g}$
\end{tabular} & 2.4 & $6-54$ & 35 & 140 & $40-120$ & Low \\
\hline \begin{tabular}{|c|} 
Wi-Fi \\
$802.11 \mathrm{n}$
\end{tabular} & $2.4 / 5$ & $2-54$ & 70 & 250 & $40-120$ & Low \\
\hline \begin{tabular}{|c|} 
DSRC \\
$802.11 \mathrm{p}$ \\
\end{tabular} & $5.8-5.9$ & $3-27$ & 30 & 1000 & $40-150$ & High \\
\hline \begin{tabular}{|c|} 
MBWA \\
802.20
\end{tabular} & 3.5 & 80 & NR & 15 & $100-250$ & High \\
\hline $\begin{array}{l}\text { WiMax } \\
802.16\end{array}$ & $\begin{array}{c}2.3,2.5 \\
3.5\end{array}$ & 1-3Gbps & NR & $\approx 50 \mathrm{~km}$ & $60-250$ & High \\
\hline \begin{tabular}{|c} 
Bluetoot \\
$\mathrm{h}$ \\
802.15 .1
\end{tabular} & $\begin{array}{c}\text { ISM } \\
\text { band } \\
2.4-2.48\end{array}$ & $1-24$ & 10 & 100 & $20-30$ & Very low \\
\hline Infrared & $\begin{array}{c}835- \\
1035 \mathrm{~nm}\end{array}$ & $1-2$ & - & 100 & 250 & Medium \\
\hline GPS & 1.575 & - & - & - & - & Medium \\
\hline Cellular & $\begin{array}{c}\text { Operator } \\
\text { Depende } \\
n t\end{array}$ & $\begin{array}{c}384 \\
\text { kbps-2 } \\
\text { Mbps }\end{array}$ & NR & 3000 & $100-50$ & High \\
\hline
\end{tabular}

In this work the author propose an efficient environmental model such Rural (R), Highway (H) and Urban (U) for V2I environment considering the environmental factor. The proposed RHU model is incorporated into the Slotted-Aloha in IEEE 802.11p MAC protocols, which served as a spine for catering Safety application and Non-Safety applications. The 802.11p also known as DSRC/WAVE technologies support low latency V2I communication.

The paper organization is as follows: The literature survey is presented in Section two. The proposed environmental model considering environmental factor are presented in Section three. The results and the experimental study are presented in the section four. The concluding remark is discussed in the last section

\section{LITERATURE SURVEY}

Every technology has some strength and weakness in terms of reliability, effectiveness, efficiency, cost, maintenance and ease of use. The primary objective of almost all the vehicular wireless technology is to provide safety. The architecture of DSRC is so 
defined that it is suitable for safety as well as non-safety applications. Various researches have been conducted on DSRC technology and confirmed its effectiveness. According to vehicular wireless technology comparison depicted in Table.1, it is evident that DSRC is most suitable in terms of cost, data transfer rate and signal range. DSRC works on Wi-Fi pattern and there are various previous researches made on802.11 based infotainment based services. Ott and Kutscher [19] [20] experimented with V2X communication using IEEE 802.11b. They found $802.11 \mathrm{~b}$ supports persistent Internet connection on slow moving vehicles with high data rate. It has the capability to intermittently connect on fast moving vehicles at the approximate speed of $40-180 \mathrm{~km} / \mathrm{h}$ with data rate suitable for applications which does not require continuous connection like sending an email message, browsing a web page etc. Sporadic connection has several intricacies in accessing applications which require continuous session like VOIP, video streaming, remote database access, file access etc. Both they empirically showed a vehicle moving at the velocity of $180 \mathrm{~km} / \mathrm{h}$ has about 10 seconds of persistent connection to single pass through AP. They managed to transmit $9 \mathrm{MB}$ of data in single pass through Connectivity Island with single AP which confirm the feasibility of internet using $802.11 \mathrm{~b}$ on moving vehicle even at the speed of $180 \mathrm{~km} / \mathrm{h}$. The most common Internet protocol suite TCP and UDP has been tested in various scenarios and found TCP outperform UDP in a moving vehicle. Though internet has successfully been tested, yet many issues remain to be addressed. In [1] Cooperative VehicleInfrastructure Systems (CVIS) equipment's are used to measure and study the effects of the 802.11p communication channel in various environments. Measurements are carried out based on the packet transacted data and the received signal strength indicator (RSSI). The results presented in the paper show the effects of the environments on Packet Loss Rate and Network throughputs.

The effects of vehicle mobility speeds are not clearly described in the research work presented in [1]. In [5] the IEEE 802.11p is compared with the IEEE 802.11 b for V2V (i.e. Vehicle to Vehicle Networks) networks in different environments. The mobility models and the simulations tools that can be used to simulate VANETS are also discussed. The results presented in this paper prove that the IEEE 802.11p is efficient and out performs the IEEE 802.11 b protocol considering safety and nonsafety applications operating under the Urban and highway environmental models. The performance comparisons are carried out considering end to end delays, delivery ratios and network throughput. Urban Environment models are complex and difficult to model. The urban model is dependent on the terrain elevations, traffic density, road layouts, vegetation and vehicle types. The study presented in [9] proves this fact. In [9] the V2I field testing results conducted in the city of Bologna, Italy is presented and the communication and measurements results are discussed. A WAVE point coordination function (WPCF) is proposed in [13] for mobility management. The paper highlights the drawbacks of the 802.11p MAC in handling high number of users and high mobility speeds [17] of vehicles in the V2I scenario. In [17] an analytical model for the throughput of the EDCA mechanism in IEEE $802.11 \mathrm{p}$ protocol is discussed. The simulation results described in the paper prove the accuracy of the analytical model developed by the authors. The results also prove that the throughput reduces when the mobility speed of the users is high. The authors in [17] have considered generic noise in the analytical model, which is not the case in the real world scenario. There is a need to develop an analytical model for the throughput of the transport layer considering urban, rural and highway conditions. In [18] the effects of user node or vehicle mobility speeds on network throughput are discussed. The research presented shows throughput degradation when the user node speed is increased. In [18] it is concluded that the user node mobility parameter is important factor to be considered in network performance analysis. Based on the literature review it is clear that the environmental conditions are critical to achieve accurate and acceptable simulation studies.

\section{PROPOSED MODEL}

The author aim to design efficient environmental model such as rural, highway and urban, so that it is well-suited with the IEEE 802.11 p protocol that is designed to accommodate the provision of wireless admission in VANET [28], [29]. The proposed environment model is incorporate in to slotted aloha [24, 37] and experiment are conducted for collision and throughput efficiency. The author is intended to design an efficient environment model for V2I.

Here the author consider different environment models such as rural, highway and urban (RHU), where vehicles moves through numerous RSUs placed alongside these environment models and gain access to internet for stipulated session. Let consider that automobile require to transmit a packet when it is in the RSUs coverage range, and pays for channel access attempts. As both the achievable bandwidth and channel contention level fluctuate over session period, the automobile needs to take decision when to transmit packet by considering channel availability, the infotainment applications quality of service necessity, and requirement level of contention in current and forthcoming session slots considering signal degradation of environment factor.

\subsection{PROPOSED RHU ENVIRONMENTAL MODEL FOR V2I}

In this work the author consider a different scenario such as urban, rural and highway environmental model for V2I, where multiple RSUs are set up and they are interconnected through support (spine) network to provide infotainment application facilities to automobiles or end user within their range of coverage. In this work the author focus on an automobile that wants to transmit a packet of size $S_{p k t}$ when it travels through a part of this RHU environment model with a set of RSUs $I=$ $(1,2, \ldots, y)$, where the vehicles pass through the $x^{\text {th }}$ RSU before the $y^{\text {th }} \mathrm{RSU}$ for $x<y$ with $x, y \in I$. The author assumes that the $y^{\text {th }} \mathrm{RSU}$ has a communication range $T R_{y}$. The author also considers that the automobile is associated to one RSU at a period. If the communication radius of the RSUs is coinciding, then appropriate handover between the RSUs will be done based on [30]. Here the author considers that the RSUs are designed in such a way that any location in this network of RHU environmental model is concealed by an RSU. The proposed model can be extended to consider the situations where the area of coverages of adjacent RSUs is inaccessible from each other. 


\subsection{RHU TRAFFIC MODELLING}

Let $\mu$ represent the average number of vehicles passing through a stationary RSU per unit time. Let us consider that the number of vehicles passing into this segment of the RHU scenario follows a Poisson method [31] with the mean arrival rate $\mu$. Let $d$ represent the density of vehicle i.e. the number of vehicles per unit distance along the RHU road segment, and $u$ be the mobility speed of the vehicles. From [32], we have,

$$
\mu=d u
$$

The context between the speed $u$ and vehicle density $d$ is represented by the following Eq.(17),

$$
u=u_{f f s}\left(1-d / d_{\max }\right)
$$

where, $u_{f f s}$ is the free-flow mobility speed of automobile on the road without any other automobile, and $d_{\max }$ is the maximum automobile load.

As we are evaluating the vehicle movement in steady state, all the automobiles within the communication range of RSU are presumed to travel with the identical speediness $u$ in Eq.(2). The floor function is represented by [.|. The maximum amount of user automobile that can be put up within the communication area of the $y^{\text {th }} \mathrm{RSU}$ is represented by,

$$
V_{\text {max }, y}=\left\lfloor 2 A_{y d \max }\right\rfloor, \forall y \in I
$$

Wireless signal radio propagations are affected from factor such as fading, shadowing, and path loss. Subsequently the communication remoteness among the RSU and the user vehicle differs in the RHU environment, due to signal degradation of path loss the author focus on the foremost effect of channel attenuation for different environmental model. The bandwidth at slot t time is represented as,

$$
b w_{t}=C_{B W} \log _{2}\left(1+\frac{T^{p}}{N_{o} C_{B W} d_{t}^{\gamma}}\right)
$$

where, $T^{p}$ is the transmission power of the user automobile, $C_{B W}$ is the network channel data rate, $d_{t}^{\gamma}$ is the distance between the closest RSU and the automobile at slot $t$ time, and $\gamma$ is the exponent of path loss.

The author considers the power spectral density $N_{o} / 2$ and zero mean Gaussian noise. The author also considers a scenario with fixed data rate. Since Lognormal shadowing path loss model provides an efficient way in finding path loss receptions compared to the other model that are available such as Rayleigh and Nakagami path loss models for wireless environments, the author have selected it as the channel model [33]. In log-normal environment shadowing path loss model, the signal to noise ratio $(d)_{\mathrm{dB}}$ at a distance from the transmitter $d$ to the receiver is represented as follows:

$$
\gamma(d)_{\mathrm{dB}}=P_{t}-P L\left(d_{o}\right)-10_{\eta} \log _{10}\left(d / d_{o}\right)-X_{\sigma}-P_{\eta}
$$

where, $P L\left(d_{o}\right)$ signifies the path loss at a reference distance $d_{o}, P_{t}$ signifies the transmission power in $\mathrm{dBm}, \eta$ means the exponential path loss, $P_{\eta}$ represents the noise power in $\mathrm{dBm}$ and $X_{\sigma}$ represents a zero mean Gaussian random variable with standard deviation $\sigma$.

Based on Eq.(5) the path loss $\gamma(d)_{\mathrm{dB}}$ obtained for rural environment is 1.79 with standard deviation equal 3.3 , for rural environment is 1.61 with standard deviation equal 3.4 and for highway environment is 1.85 with standard deviation equal 3.2. Based on these path loss values for different environment model $\gamma$ the bandwidth of the destination vehicle is estimated using Eq.(4) and thus improving throughput efficiency and reducing packet collision of our model.

The proposed RHU environmental model with bears the closest similarity to our work and is used for comparisons in the

\begin{tabular}{|c|c|c|c|c|c|}
\hline & $\begin{array}{c}\text { RHU- } \\
\text { EM }\end{array}$ & $\begin{array}{c}\text { MS- } \\
\text { ALOHA } \\
{[34]}\end{array}$ & $\begin{array}{c}\text { CR- } \\
\text { VANET } \\
{[35]}\end{array}$ & $\begin{array}{c}\text { SLOP } \\
{[37]}\end{array}$ & $\begin{array}{c}\text { ZCOR } \\
{[38]}\end{array}$ \\
\hline $\begin{array}{c}\text { Environ } \\
\text { mental } \\
\text { model }\end{array}$ & RHU & $\begin{array}{l}\text { urban and } \\
\text { highway }\end{array}$ & $\begin{array}{l}\text { Urban } \\
\text { street }\end{array}$ & $\begin{array}{c}\text { intellig } \\
\text { ent } \\
\text { driver }\end{array}$ & $\begin{array}{l}\text { Monte } \\
\text { Carlo }\end{array}$ \\
\hline $\begin{array}{l}\text { Scheduli } \\
\text { ng } \\
\text { Algorith } \\
\text { m }\end{array}$ & $\begin{array}{c}\text { Slotted } \\
\text { aloha }\end{array}$ & $\begin{array}{c}\text { MS- } \\
\text { ALOHA }\end{array}$ & $\begin{array}{l}\text { Slotted } \\
\text { aloha }\end{array}$ & $\begin{array}{l}\text { Wave- } \\
\text { Slotted } \\
\text { aloha }\end{array}$ & $\begin{array}{c}\text { R- } \\
\text { ALOHA }\end{array}$ \\
\hline $\begin{array}{c}\text { Simulato } \\
\mathrm{r}\end{array}$ & SIMITS & VISSIM & Yes (NA) & $\begin{array}{l}\text { YES } \\
\text { (NA) }\end{array}$ & $\begin{array}{c}\text { NS-2 } \\
\text { simulatio } \\
n\end{array}$ \\
\hline MAC & $\begin{array}{c}802.11 \mathrm{p} \\
\text { MAC }\end{array}$ & $\begin{array}{c}802.11 \mathrm{p} \\
\text { MAC }\end{array}$ & $\begin{array}{l}\text { Uniform } \\
\text { MAC[36] }\end{array}$ & $\begin{array}{c}802.11 p \\
\text { MAC }\end{array}$ & $\begin{array}{c}802.11 \mathrm{e} \\
\text { MAC }\end{array}$ \\
\hline
\end{tabular}
experimental study presented here. The comparison of proposed RHU environmental model with its counterparts is summarized in Table.2.

Table.2. Comparison with other work

\section{RESULTS AND ANALYSIS}

The system environment used is windows 8.1 64-bit operating system with $6 \mathrm{~GB}$ of ram. The author have used dot net framework 4.0 and $\mathrm{C \#} 6.0$ programming language for the proposed work and conducted experimental study on following parameter for throughput efficiency and packet collision for varied number of vehicle users and evaluate the performance of varied environmental model. The authors have considered IEEE 802.11p also known as DSRC/WAVE which has six services channel and one control channel and S-ALOHA as MAC scheduling algorithm. The modulation scheme used is QPSK which has as a transfer rate of $9 \mathrm{Mbps}$ (Megabits per seconds) with coding rate of 0.75 and in experiment 4.1 the number of vehicle is varied to 10 , 20, 30 and 40 and in experiment 4.2 the mobility speed (it represent number of frames per cycle) is varied 3, 6 and 9 (where 3 represent the maximum speed and 9 represent the minimum speed) and the number of vehicle is fixed to 40 and simulation is conducted for Rural (R), Highway (H) and Urban (U) environmental model.

\subsection{COLLISION, SUCCESSFUL TRANSMISSION AND THROUGHPUT EFFICIENCY CONSIDERING VARIED VEHICLE DENSITY FOR RHU ENVIRONMENTAL MODEL}

In Fig.2, the throughput efficiency for varied user in highway environmental model. The Fig. 3 shows the throughput efficiency for varied user in rural environmental model. The Fig. 4 shows the throughput efficiency for varied user in urban environmental model. From all three-environment models in Fig.2, Fig.3 and 
Fig. 4 it can be seen that throughput achieved is increased when the number of user vehicle is increased.

The Fig. 5 shows the average throughput achieved by varying user vehicle and its impact on throughput for RHU environmental model. In Fig.5, it shows that the throughput achieved in urban environment is high when compared to highway and rural model and the throughput achieved in the rural is low when compared to urban and highway model. The average throughput achieved for 10 users for highway environment is about $2.31 \mathrm{Mbps}$, for rural environment is about $1.52 \mathrm{Mbps}$ and for urban environment is about $2.68 \mathrm{Mbps}$. The average throughput achieved for 20 users for highway environment is about $4.12 \mathrm{Mbps}$, for rural environment is about $3.26 \mathrm{Mbps}$ and for urban environment is about 4.42Mbps. The average throughput achieved for 30 users for highway environment is about $4.82 \mathrm{Mbps}$, for rural environment is about $4.44 \mathrm{Mbps}$ and for urban environment is about 5.67Mbps. The average throughput achieved for 40 users for highway environment is about $5.18 \mathrm{Mbps}$, for rural environment is about $4.72 \mathrm{Mbps}$ and for urban environment is about 6.11Mbps.

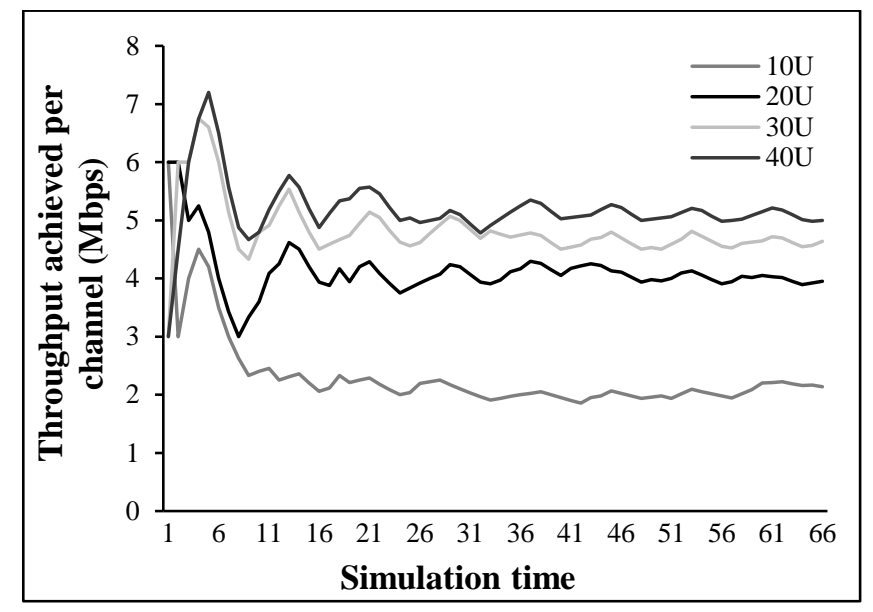

Fig.2. Throughput achieved per Channel for varied user in highway environmental model

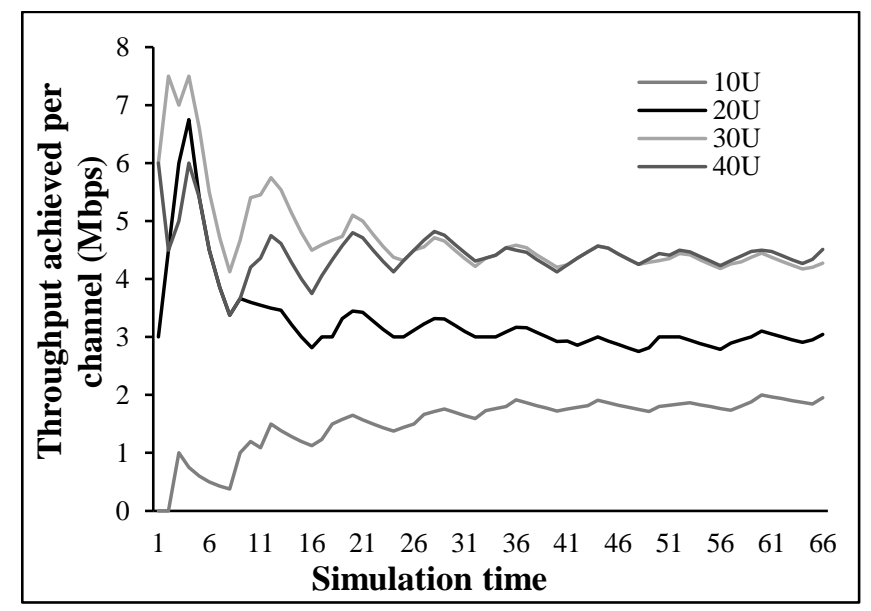

Fig.3. Throughput achieved per Channel for varied user in rural environmental model

In Fig.6, the number of packet transmitted successfully between V2I. The packet transmitted successfully is high in urban model when compared to highway and rural model and packet transmitted successfully is low in rural model when compared to highway and urban model and also we can see that the packet transmission increases with increasing number of users.

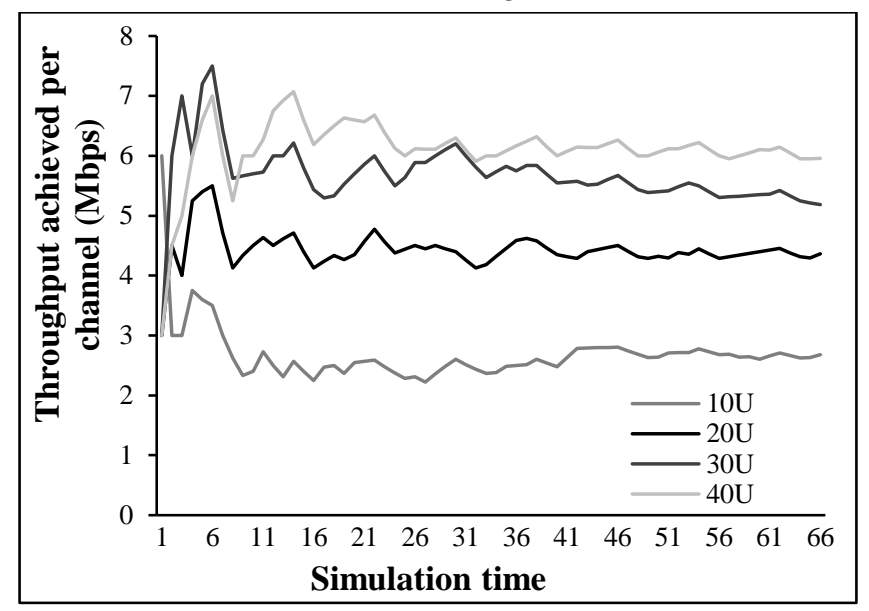

Fig.4. Throughput achieved per Channel for varied user in urban environmental model

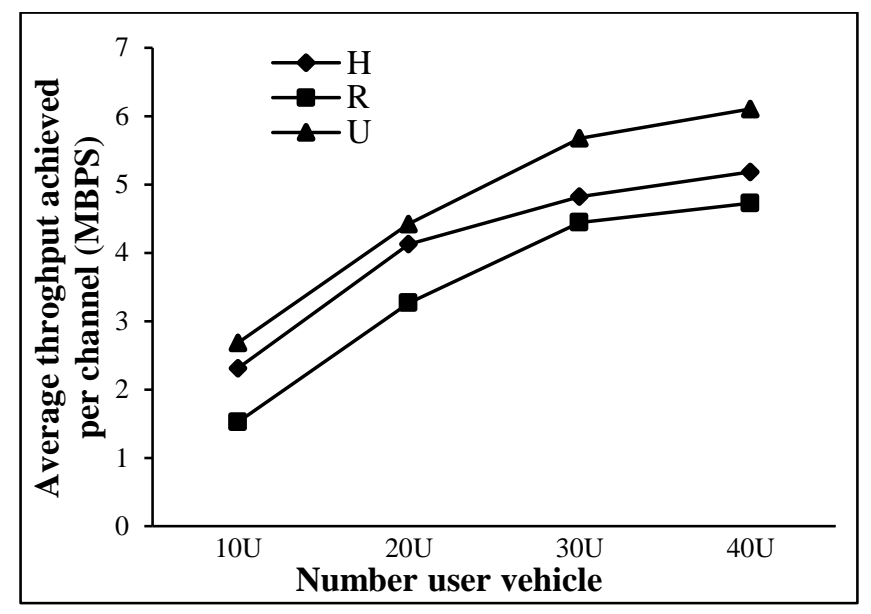

Fig.5. Average throughput achieved per cycle for varied vehicle user

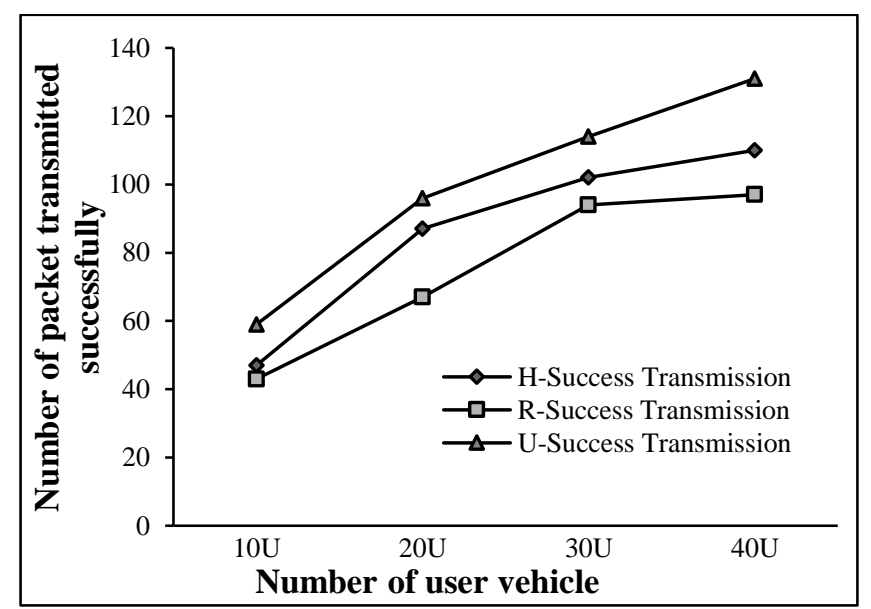

Fig.6. Packet transmitted successfully for varied vehicle user

In Fig.7, the number of packet collision in V2I. The packet collision is high in rural model when compared to highway and urban model, packet collision is low in urban model when 
compared to highway, and rural model and also we can see that the packet collision increases with increasing number of users.

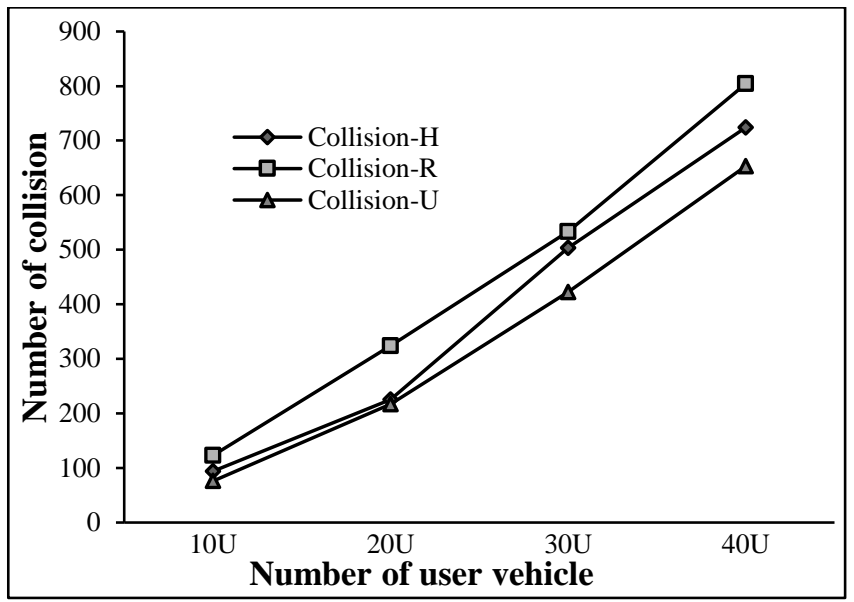

Fig.7. Packet collision for varied user

\subsection{COLLISION, SUCCESSFUL TRANSMISSION AND THROUGHPUT EFFICIENCY CONSIDERING VARIED MOBILITY SPEED FOR RHU ENVIRONMENTAL MODEL}

In Fig. 8, the average throughput achieved by varying vehicle mobility (cycle per frame) speed and its impact on throughput for RHU environmental model. In Fig.8, it shows that the average throughput achieved in urban environment is high when compared to highway and rural model and the average throughput achieved in the rural is low when compared to urban and highway model. The average throughput for achieved 3 cycles per frame for highway environment is about $4.758 \mathrm{Mbps}$, for rural environment is about $4.37 \mathrm{Mbps}$ and for urban environment is about 5.814Mbps.

The average throughput achieved for 6 cycles per frame for highway environment is about $4.988 \mathrm{Mbps}$, for rural environment is about $4.523 \mathrm{Mbps}$ and for urban environment is about $5.869 \mathrm{Mbps}$. The average throughput achieved for 9 cycles per frame for highway environment is about $5.389 \mathrm{Mbps}$, for rural environment is about $5.001 \mathrm{Mbps}$ and for urban environment is about $6.283 \mathrm{Mbps}$. From simulation result obtain we can see that throughput efficiency depend on mobility speed of user vehicle as we can see when speed is maximum ( 3 cycle per frame) the throughput achieved is low and when speed is minimum $(9$ cycle per frame) the throughput achieved is high for all RHU environment model which is shown in Fig.8.

In Fig.9, the number of packet transmitted successfully between V2I for varied cycle per frame (mobility). The packet transmitted successfully is high in urban model when compared to highway and rural model and packet transmitted successfully is low in rural model when compared to highway and urban model and we can see that the packet transmission increases with increasing in mobility speed.

In Fig.10, the number of packet collision in V2I for varied cycle per frame (mobility). The packet collision is high in rural model when compared to highway and urban model and packet collision is low in urban model when compared to highway and rural model and also we can see that the packet collision increases with increasing mobility speed.

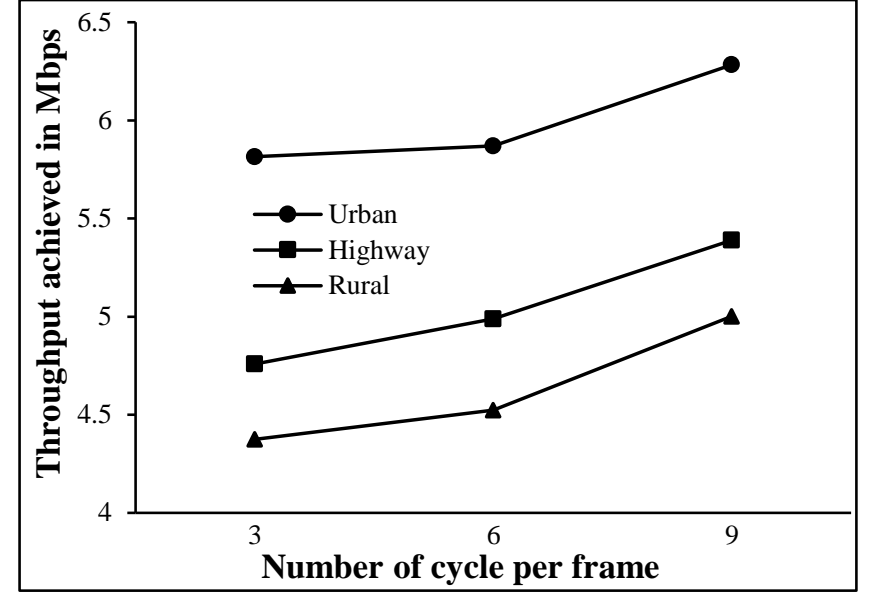

Fig.8. Average throughput achieved for varied mobility speed

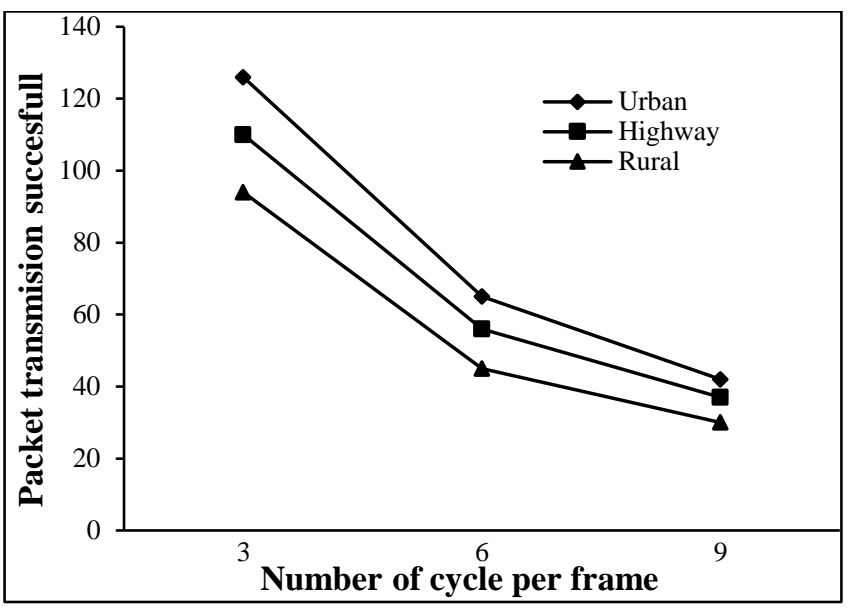

Fig.9. Packet transmission successful for varied mobility speed

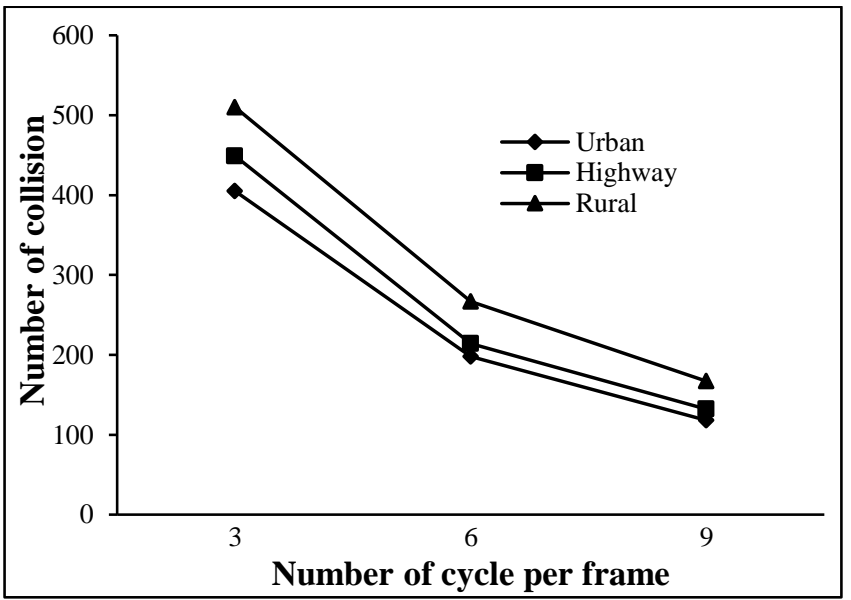

Fig.10. Packet collision achieved for varied mobility speed

\section{CONCLUSION}

The SITS has been lately engrossed by industry as well as academic world since it has the prospective to save environment, money, lives and travel time. A VANET is a vital element of ITS which uses various communication standards in order to provision services for V2I and V2V. In VANET choosing the right communication standard is an important factor since it defines the 
data rate, range of transmission and secureness. The existing scheduling technique does not consider the environmental factor which affects the throughput performance and increases packet drop rate which intern incur packet delay. Here in this work the author propose an efficient V2I environment model considering the environmental factor. The efficient environmental model algorithm is incorporated into slotted aloha in IEEE $802.11 \mathrm{p}$ MAC protocols which served as a spine for catering safety application and non-Safety applications.

The 802.11p also known as DSRC/WAVE technologies support low latency V2I communication. Experiments are conducted for collision and throughput efficiency for different environment model such as rural, urban and highway and also by varying traffic load and by varying mobility of user vehicle. The author studied impact of speed and traffic load on V2I scenario on throughput efficiency and collision which is an important factor of V2I application services. The experimental result shows the proposed environmental model for S-ALOHA and its impact on collision and throughput efficiency and thus helps in improving QoS for VANET application. In future work The author propose a new novel adaptive mac scheduler for varied environmental model proposed in this work to reduce the collision which is an issue in S-ALOHA and improve the throughput efficiency by considering various OFDM modulation scheme such BPSK and QAM that are supported for IEEE 802.11p Protocol.

\section{REFERENCES}

[1] P. Keeratiwintakorn, E. Thepnorarat and A. Russameesawang, "Ubiquitous Communication for V2V and V2I for Thailand Intelligent Transportation System", Proceedings of NTC International Conference, pp. 1-6, 2009.

[2] Transparency Market Research, "Connected Car MarketGlobal Industry Analysis, Size, Share, Growth, Trends and Forecast, 2013-2019”, Available at: http://www.transparencymarketresearch.com/connectedcar.html.

[3] S. Al-Sultan, M.M. Al-Doori, A.H. Al-Bayatti and H. Zedan, "A Comprehensive Survey on Vehicular Ad-Hoc Network", Journal of Network and Computer Applications, Vol. 37, pp. 380-392, 2014.

[4] D. Schrank, B. Eisele and T. Lomax, “TTI's 2012 Urban Mobility Report", Texas A\&M Transport Institution, Texas A\&M University, pp. 1-63, 2012.

[5] S. Yousefi, T. Chahed, S.M.M. Langari and K. Zayer, "Comfort Applications in Vehicular Ad Hoc Networks based on Fountain Coding", Proceedings of Vehicular Technology Conference, pp. 1-5, 2010.

[6] J. Anda, J. LeBrun, D. Ghosal, C.N. Chuah and M. Zhang, "VGrid: Vehicular Adhoc Networking and Computing Grid for Intelligent Traffic Control", Proceedings of IEEE 61 ${ }^{\text {st }}$ Vehicular Technology Conference, pp. 2905-2909, 2005.

[7] P. Pereira, A. Casaca, J. Rodrigues, V. Soares, J. Triay and C. Cervello-Pastor, "From Delay-Tolerant Networks to Vehicular Delay-Tolerant Networks", IEEE Communications Surveys and Tutorials, Vol. 14, No. 4, pp. 1166-1182, 2012.
[8] C. Olaverri-Monreal, P. Gomes, R. Fernandes, F. Vieira and M. Ferreira, "The See-Through System: A VANET-Enabled Assistant for Overtaking Maneuvers", Proceedings of IEEE Intelligent Vehicle Symposium, pp. 123-128, 2010.

[9] G. Dimitrakopoulos, "Intelligent Transportation Systems based on Internet-Connected Vehicles: Fundamental Research Areas and Challenges", Proceedings of $11^{\text {th }}$ International Conference on ITS Telecommunications, pp. 145-151, 2011.

[10] A. Balasubramanian, R. Mahajan, A. Venkataramani, B.N. Levine and J. Zahorjan, "Interactive Wi-Fi Connectivity for moving Vehicles", ACM Computer Communication Review, Vol. 38, No. 4, pp. 427-438, 2008.

[11] M.L. Sichitiu, N.C. Raleigh and M. Kihl, "Inter-Vehicle Communication Systems: A Survey", IEEE Communications Surveys and Tutorials, Vol. 10, pp. 88105, 2008.

[12] J. Lin, S. Chen, Y. Shih and S. Chen, "A Study on Remote On-Line Diagnostic System for Vehicles by Integrating the Technology of OBD, GPS, and 3G", World Academy Science Engineering and Technology, Vol. 56, pp. 56-59, 2009.

[13] Jingbang Wu, Huimei Lu and Yong Xiang, "Measurement and Comparison of Sub-1GHz and IEEE 802.11p in Vehicular Networks", Proceedings of IEEE Symposium on Computers and Communications, pp. 1063-1066, 2017.

[14] M. Ramadan, M. Al-Khedher and S. Al-Kheder, "Intelligent Anti-Theft and Tracking System for Automobiles", International Journal of Machine Learning and Computing, Vol. 2, No. 1, pp. 88-92, 2012.

[15] H. Hartenstein and K.P. Laberteaux, "A Tutorial Survey on Vehicular Ad Hoc Networks", IEEE Communications Magazine, Vol. 46, pp. 164-171, 2008.

[16] M.A. Abakar, R.A. Saeed, A.A. Hassan, O.M. Mohammed, O. Khalifa and S. Islam, "The Challenges of Wireless Internet Access in Vehicular Environments", Proceedings of Information and Communication Technology for the Muslim World, pp. 31-36, 2010.

[17] John B. Kenney, "Dedicated Short-Range Communications (DSRC) Standards in the United States", Proceedings of the IEEE, Vol. 99, No. 7, pp. 1162-1182, 2011.

[18] B. Haider, S. Henna, A. Gul and F. Aadil, "A Survey on Mobility Management Techniques in VANETs", Proceedings of IEEE International Conference on Computer and Information Technology, pp. 125-133, 2016.

[19] J. Ott and D. Kutscher, "The Drive-thru Architecture: WLAN-based Internet Access on the Road", Proceedings of IEEE 59 $9^{\text {th }}$ Vehicular Technology Conference, pp. 15-19, 2004.

[20] J. Ott and D. Kutscher, "Internet Access for Mobile Users: From Drive-Thru Internet to Delay-Tolerant Ad-Hoc Networking", Proceedings of Multi-Hop Ad Hoc Network Theory, pp. 241-247, 2007.

[21] F. Bai, D.D. Stancil and H. Krishnan, "Toward Understanding Characteristics of Dedicated Short Range Communications (DSRC) from a Perspective of Vehicular Network Engineers", Proceedings of $16^{\text {th }}$ Annual International Conference on Mobile Computing and Networking, pp. 329-340, 2010. 
[22] R. Gass, J. Scott and C. Diot, "Measurements of In-Motion 802.11 Networking", Proceedings of $7^{\text {th }}$ IEEE Workshop on Mobile Computing Systems and Applications, pp. 69-74, 2005.

[23] OECD Broadband Statistics to December 2006, Available at: http://www.oecd.org/sti/broadband/oecdbroadbandstatistics todecember2006.htm.

[24] F. Han, D. Miyamoto and Y. Wakahara, "RTOB: A TDMAbased MAC Protocol to Achieve High Reliability of OneHop Broadcast in VANET", Proceedings of International Conference on Pervasive Computing and Communications, pp. 87-92, 2015.

[25] I. Gaspard and G. Zimmermann, "Investigations for Broadband Internet within High-Speed Trains", Advances in Radio Science, Vol. 3, pp. 247-252, 2005.

[26] T. Van Leeuwen, I. Moerman, H. Rogier, B. Dhoedt, D. De Zutter, and P. Demeester, "Broadband Wireless Communication in Vehicles", Journal Communication Network, Vol. 2, No. 3, pp. 77-82, 2003.

[27] S. Cespedes and X. Shen, "Enabling Relay-Aided IP communications in $802.11 \mathrm{p} /$ Wave Networks", Proceedings of Global Communications Conference, pp. 5567-5572, 2012.

[28] Wireless LAN Medium Access Control (MAC) and Physical Layer (PHY) Specification, Available at: http://www.disrv.unisa.it/ vitsca/RC-0809I/IEEE-802-11.pdf.

[29] R.A. Uzcategui and G. Acosta-Marum, "Wave: A Tutorial", IEEE Communications Magazine, Vol. 47, No. 5, pp. 126133, 2009.

[30] J. Choi and H. Lee, "Supporting Handover in an IEEE 802.11p-based Wireless Access System", Proceedings of ACM International Workshop on VANETs, pp. 75-80, 2010.
[31] T. Juhana and W. Yogiyana, "Semi Uniform Segmentation Technique in Headway Model on Vehicles Communication", Proceedings of $2^{\text {nd }}$ International Conference on Wireless and Telematics, pp. 146-152, 2016.

[32] J.D. Fricker and R.K. Whitford, "Fundamentals of Transportation Engineering: A Multimodal Systems Approach", Prentice Hall, 2004.

[33] S.A. Vaqar and O. Basir, "Traffic Pattern Detection in a Partially Deployed Vehicular Ad Hoc Network of Vehicles", IEEE Wireless Communications, Vol. 16, No. 6, pp. 40-46, 2009.

[34] A. Bazzi, A. Zanella and B.M. Masini, "An OFDMA-based MAC Protocol for Next-Generation VANETs", IEEE Transactions on Vehicular Technology, Vol. 64, No. 9, pp. 4088-4100, 2015

[35] Nan Cheng, Ning Zhang, Ning Lu, Xuemin Shen, J.W. Mark and Fuqiang Liu, "Opportunistic Spectrum Access for CRVANETs: A Game-Theoretic Approach", IEEE Transactions on Vehicular Technology, Vol. 63, No. 1, pp. 237-251, 2014.

[36] L.M. Law, J. Huang and M. Liu, "Price of Anarchy of Wireless Congestion Games", IEEE Transactions on Wireless Communications, Vol. 11, No. 10, pp. 3778-3787, 2012.

[37] Nuno Fabio G.C. Ferreira and Jose A.G. Fonseca, "Improving Safety Message Delivery through RSU's Coordination in Vehicular Networks", Proceedings of IEEE World Conference on Factory Communication Systems, pp. 1-8, 2015.

[38] S. Oh, M. Gruteser and D. Pompili, "Coordination-Free Safety Messages Dissemination Protocol for Vehicular Networks", IEEE Transactions on Vehicular Technology, Vol. 16, No. 9, pp. 1-13, 2011. 